\title{
Turbulent Stress Invariant Analysis: Clarification of Existing Terminology
}

\author{
A.J. Simonsen ${ }^{1}$ and P.-Å. Krogstad ${ }^{2}$ \\ ${ }^{1}$ Present address: SINTEF Energy Research, Energy Processes. Kolbjørn Hejes v. 1b, N-7065 Trondheim, Norway \\ ${ }^{2}$ Department of Energy and Process Engineering, The Norwegian University of Science and Technology, N-7491 \\ Trondheim, Norway
}

\begin{abstract}
As a means of studying the structure of the turbulence, the time mean invariants, defined according to the theory of Lumley [3] has proven to be a useful and popular tool. According to the theory there is a domain, known as the Lumley triangle, within which all realizable Reynolds's stress invariants must lie. The borders of this domain describes different states of the turbulent stress tensor. It has been found that there is some confusion in the terminology used when describing these states. The confusion is related to whether the notation is used to describe the shape of the stress tensor or the eddies of the turbulence. Choi and Lumley [1] noted the same controversy in terminology, but since the confusion is still found to exist the current work describes the fundamental relationships which exist between the shape of the stress tensor and it's invariants. Also an analysis is given to clarify the existing terminology.
\end{abstract}

\section{Introduction}

Any symmetric tensor, like the stress tensor $\tau_{i j}$, may be divided into a traceless deviator, $\tau_{i j}^{\prime}$ and an isotropic part, $\tau_{i j}^{\circ}$

$$
\tau_{i j}=\tau_{i j}^{\circ}+\tau_{i j}^{\prime}
$$

where

$$
\tau^{\circ}=\frac{1}{3} \tau_{k k} \delta_{i j}
$$

and

$$
\tau_{i j}^{\prime}=\tau_{i j}-\frac{1}{3} \tau_{k k} \delta_{i j}
$$

In the case of true isotropy all the elements in $\tau_{i j}^{\prime}$ would become zero. The non-dimensional form of the anisotropy tensor is given by

$$
b_{i j}=\frac{\tau_{i j}^{\prime}}{\tau_{k k}}=\frac{\tau_{i j}}{\tau_{k k}}-\frac{1}{3} \delta_{i j}
$$

By using the Cayley-Hamilton theorem, the characteristic equation for any second order symmetric tensor may be written as

$$
\sigma^{3}-I_{1} \sigma^{2}+I_{2} \sigma-I_{3}=0
$$

where the $I$ 's are the first, second, and third invariants of the tensor and $\sigma$ is the principal stress. The invariants are related to the tensor terms according to the relations

$$
\begin{aligned}
& I_{1}=b_{k k} \\
& I_{2}=-\frac{1}{2} b_{i j} b_{j i} \\
& I_{3}=\operatorname{det}\left(b_{i j}\right)
\end{aligned}
$$

where the flow has been assumed to be incompressible.
Assuming incompressible axisymmetric turbulence with $y$ as the symmetry axis, and using cylindrical coordinates, the invariants may be expressed as

$$
\begin{aligned}
& I_{1}=0 \\
& I_{2}=-\frac{1}{2}\left[b_{r r}^{2}+b_{y y}^{2}+b_{\theta \theta}^{2}+2 b_{r y}^{2}\right] \\
& I_{3}=\left[b_{r r} b_{y y}-b_{r y}^{2}\right] b_{\theta \theta}
\end{aligned}
$$

In solving the characteristic equation, equation (5), for a given set of invariants the eigenvalues and the related eigenvectors of the tensor are revealed. This gives the main stress directions of the stress tensor and their magnitude. Several solutions are possible depending on the stress field, but there are also definitive bounds with respect to the realizability of the flow which have led to the anisotropy invariant map (initially proposed by Lumley [3]) and therefore often referred to as the Lumley triangle) given in figure 1.

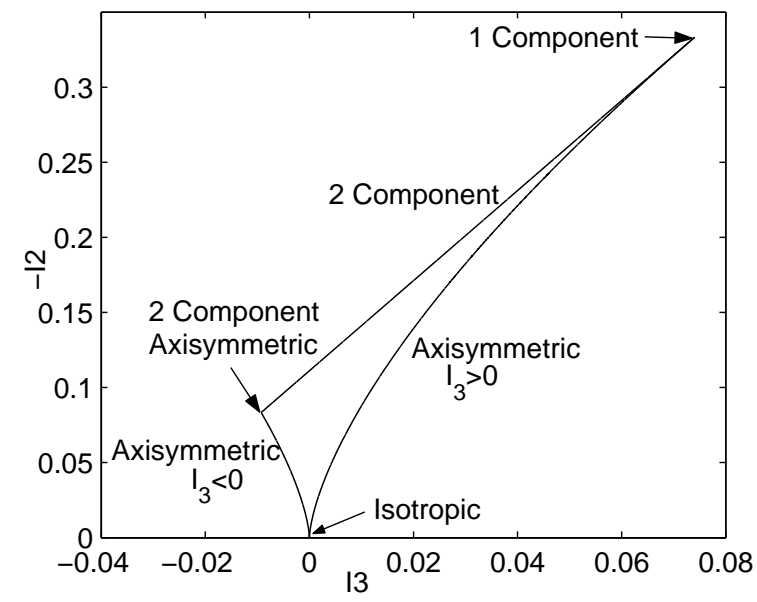

Figure 1: The anisotropy invariant map, AIM.

At the origin $\left(I_{2}=I_{3}\right)$ the isotropic state is found. From this point two limiting lines are found where the flow is assumed to be axisymmetric (i.e. $b_{r y}=0$ and two of the diagonal elements are equal e.g. $\left.b_{r r}=b_{\theta \theta}\right)$. This leads to the relation $I_{3}= \pm 2\left(-I_{2} / 3\right)^{3 / 2}$. Each of these lines have a limiting value. In the first case the two identical diagonal terms are smaller than the third element and tend to zero. This leads to a 1 component state of turbulence (1C in figure 1). The other possibility is that the two identical elements dominate so that the third component is ignorable. This leads to the two component axisymmetric state indicated in figure 1 . The line joining 1- and 2-component axisymmetric turbulence represents the all other possible states where only two diagonal components exist.

As a tool in describing the state of the turbulence Lee and 
Reynolds[2] designated different shapes of the stress field to the value of the invariants in equation (5) such as rod and disk like turbulence. However, in their description Lee and Reynolds were focusing on the turbulent eddies rather than the stress field itself. This has over the years lead to a confusion when describing the stress field since the characteristic shapes related to the stresses and the eddies are not generally the same. If not aware of this the axisymmetric limits of the Lumley triangle will be described by shapes that do not relate to the stress tensor the invariants represent.

In the following the relations between the stress field and the invariants are shown and then related to the shape of the stress tensor. This will be compared to the shape of the turbulent eddies for the same type of turbulence. It is must be emphasized that this analysis does not have an influence on turbulence modelling, but have implications in understanding and visualization of the stress field. It must also to be noted that the aim of the current paper is to clarify the terminology used.

\section{The characteristic shape related to the stress tensor}

In terms of the stress tensor the visualization of the stress field is determined by the relative sizes of the three main stresses and the designations of the axisymmetric limits are based upon the following analysis. The Reynolds stress tensor, $\tau_{i j}$, is a second order symmetric tensor. As stated by the spectral theorem every real symmetric matrix can be diagonalized by an orthonormal matrix. In other words the Reynolds stress matrix,

$$
\mathbf{T}=\tau_{i j}=\left(\begin{array}{lll}
\tau_{11} & \tau_{12} & \tau_{13} \\
\tau_{21} & \tau_{22} & \tau_{23} \\
\tau_{31} & \tau_{32} & \tau_{33}
\end{array}\right)
$$

may be written as

$$
\mathbf{T}=\mathbf{Q} \mathbf{\Sigma} \mathbf{Q}^{-1}
$$

where

$$
\boldsymbol{\Sigma}=\left(\begin{array}{ccc}
\sigma_{1} & 0 & 0 \\
0 & \sigma_{2} & 0 \\
0 & 0 & \sigma_{3}
\end{array}\right)
$$

is the eigenvalue matrix of $\mathbf{T}$ and $\sigma_{i}$ are the eigenvalues. $\mathbf{Q}$ is an orthonormal matrix with the properties

$$
\mathbf{Q} \mathbf{Q}^{-1}=\mathbf{Q} \mathbf{Q}^{T}=\mathbf{I} \text {. }
$$

Now, recalling that the stress, $\mathbf{f}_{\tau}$, in the direction of a vector $\mathbf{n}$ is represented by the linear operator $\mathbf{T}$ on $\mathbf{R}^{\mathbf{3}}\left(\mathbf{T}: \mathbf{R}^{\mathbf{3}} \rightarrow \mathbf{R}^{\mathbf{3}}\right.$ where both $\mathbf{n}, \mathbf{f}_{\tau} \in \mathbf{R}^{\mathbf{3}}$ ):

$$
\mathbf{f}_{\tau}=\mathbf{T} \cdot \mathbf{n}
$$

By the use of relation 9 and left-multiplying with $\mathbf{Q}^{-1}$ this may be rewritten as

$$
\mathbf{y}=\mathbf{Q}^{-1} \mathbf{f}_{\tau}=\boldsymbol{\Sigma} \mathbf{Q}^{-1} \mathbf{n}=\boldsymbol{\Sigma} \mathbf{x}
$$

which means that by changing the old coordinate system into a new coordinate system, coinciding with the principal axis of the stress tensor, $\mathbf{T}$ becomes a diagonal matrix $\boldsymbol{\Sigma}$ with the principal stresses of $\mathbf{T}$ on its diagonal.

If $\mathbf{x}$ is considered as the normalized stress vector of an isotropic field describing the unit sphere we have

$$
\begin{aligned}
\mathbf{x} & =\left\langle x_{1}, x_{2}, x_{3}\right\rangle \\
\mathbf{x}^{T} \mathbf{x}=1 & \Leftrightarrow x_{1}^{2}+x_{2}^{2}+x_{3}^{2}=1 .
\end{aligned}
$$

Equation (12) is now representing a distortion of this stress vector due to the mapping $\mathbf{T}$. Left-multiplying equation (12) with $\Sigma^{-1}$ gives

$$
\mathbf{x}=\boldsymbol{\Sigma}^{-1} \mathbf{y}
$$

By using the equation (10) and the matrix relation

$$
(\mathbf{A B})^{T}=\mathbf{B}^{T} \mathbf{A}^{T}
$$

the following is obtained

$$
\mathbf{x}^{T} \mathbf{x}=\mathbf{y}^{T} \Sigma^{-2} \mathbf{y}=1
$$

or

$$
\left(\frac{y_{1}}{\sigma_{1}}\right)^{2}+\left(\frac{y_{2}}{\sigma_{2}}\right)^{2}+\left(\frac{y_{3}}{\sigma_{3}}\right)^{2}=1
$$

where relation 13 is used. This describes the shape of a spheroid in the coordinate system $\left(y_{1}, y_{2}, y_{3}\right)$.

In order to establish a relationship between the shape of the stress tensor and the invariants of the anisotropy tensor we need to relate the eigenvalues of $\mathbf{b}=\left(\frac{\mathbf{T}}{T_{k k}}-\frac{1}{3} \mathbf{I}\right)$ and $\mathbf{T}$. From matrix theory we know that if for two matrices $\mathbf{A}$ and $\mathbf{B}$

$$
\mathbf{A B}=\mathbf{B A}
$$

then the matrices $\mathbf{A}$ and $\mathbf{B}$ share the same eigenvectors. Since the tensor $1 / 3 \delta_{i j}$ is equal to the identity matrix $\mathbf{I} / 3$, relation 18 always holds between the matrices $\mathbf{T}$ and $\mathbf{I}$. They therefore share the same eigenvector $\mathbf{n}$ and the following relationship will hold.

$$
\mathbf{b n}=\left(\frac{\mathbf{T}}{T_{k k}}-\frac{1}{3} \mathbf{I}\right) \mathbf{n}
$$

The relationship between the eigenvalues of $\mathbf{b}$ and $\mathbf{T}$ then becomes

$$
\lambda_{i}=\frac{\sigma_{i}}{T_{k k}}-\frac{1}{3}
$$

For the non-dimensional anisotropy tensor, $\mathbf{b}$, related shapes of the ellipsoid formed by the Reynolds stresses are illustrated in figure 2 and characteristics of the flow are given in table 1 .
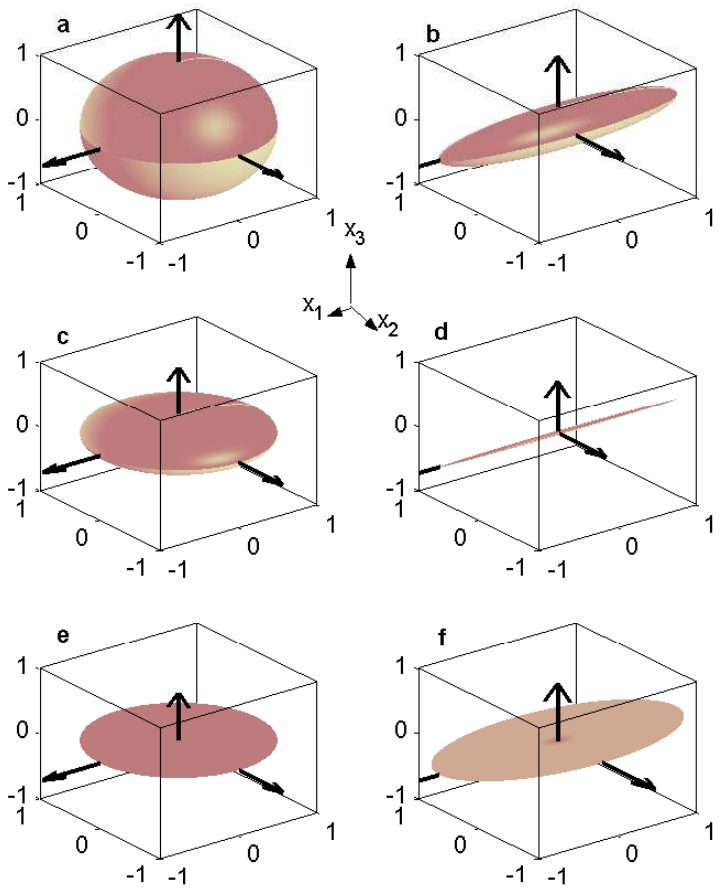

Figure 2: Illustration of the ellipsoid shapes formed by the Reynolds stress tensor in different regions of the flow. 


\begin{tabular}{|c|c|c|c|c|}
\hline $\begin{array}{l}\text { State } \\
\text { of } \\
\text { turbulence }\end{array}$ & Invariants & Eigenvalues of $b_{i j}$ & $\begin{array}{l}\text { Shape of stress } \\
\text { tensor } \\
\text { (see eq. 17) }\end{array}$ & $\begin{array}{l}\text { Symbol in } \\
\text { figures } 1 \text { and } 2\end{array}$ \\
\hline Isotropic & $I_{2}=I_{3}=0$ & $\lambda_{i}=0$ & Sphere & $\begin{array}{l}\text { a, } \\
\text { isotropic }\end{array}$ \\
\hline Axi.sym. (One large $\lambda)$ & $-\frac{I_{2}}{3}=\left(\frac{I_{3}}{2}\right)^{2 / 3}$ & $0<\lambda_{1}<\frac{1}{3},-\frac{1}{6}<\lambda_{2}=\lambda_{3}<0$ & $\begin{array}{l}\text { Prolate } \\
\text { spheroid }\end{array}$ & $\begin{array}{l}\mathrm{b}, \\
\text { axisymmetric } \\
\left(I_{3}>0\right)\end{array}$ \\
\hline Axi.sym. (One small $\lambda$ ) & $-\frac{I_{2}}{3}=\left(\frac{-I_{3}}{2}\right)^{2 / 3}$ & $-\frac{1}{3}<\lambda_{2}<0,0<\lambda_{1}=\lambda_{3}<\frac{1}{6}$ & Oblate spheroid & $\begin{array}{l}\mathrm{c}, \\
\text { axisymmetric } \\
\left(I_{3}<0\right)\end{array}$ \\
\hline One-comp. & $\begin{array}{l}I_{3}=\frac{2}{27} \\
I_{2}=-\frac{1}{3}\end{array}$ & $\lambda_{1}=\frac{2}{3}, \lambda_{2}=\lambda_{3}=-\frac{1}{3}$ & Line & $\begin{array}{l}\mathrm{d}, \\
1 \text { Component }\end{array}$ \\
\hline Two comp., axisym. & $\begin{array}{l}I_{3}=-\frac{1}{108}, \\
I_{2}=-\frac{1}{12}\end{array}$ & $\lambda_{1}=\lambda_{3}=\frac{1}{6}, \lambda_{2}=-\frac{1}{3}$ & Disk & $\begin{array}{l}\text { e, } \\
2 \text { Component, } \\
\text { (axisymmetric) }\end{array}$ \\
\hline Two component & $-I_{2}=3\left(\frac{1}{27}+I_{3}\right)$ & $\lambda_{1}+\lambda_{3}=\frac{1}{3}, \lambda_{2}=-\frac{1}{3}$ & Ellipsoid & f, \\
\hline
\end{tabular}

Table 1: Characteristics of the turbulence stress tensor and anisotropic tensor.

\section{Clarification of Terminology}

Now it is time to address the misconception encountered in the designation of the limits of Lumley's invariant map. Axisymmetric turbulence means that two of the principal stresses $\sigma_{i}$, or $\lambda_{i}$, are equal. Writing $\mathbf{b}$ in terms of the principal direction we have

$$
\mathbf{b}=\left(\begin{array}{ccc}
\lambda_{1} & 0 & 0 \\
0 & \lambda_{2} & 0 \\
0 & 0 & \lambda_{3}
\end{array}\right)
$$

and for axisymmetric turbulence we have e.g. $\lambda_{2}=\lambda_{3}$. Together with relationship $8, \lambda_{1}+\lambda_{2}+\lambda_{3}=0$, this gives for the invariants $I_{2}$ and $I_{3}$

$$
I_{2}=-\frac{3}{4} \lambda_{1}^{2} \quad, \quad I_{3}=\frac{1}{4} \lambda_{1}^{3}
$$

From the previous analysis it is clear that if we have one large principal value $\sigma_{1}$ and two equal but smaller principal values $\sigma_{2}=\sigma_{3}$, the turbulence field will be stretched in the $\mathbf{n}_{\sigma_{1}-}$ direction creating the prolate spheroid of figure $2 \mathrm{~b}$ ). On the other hand, if $\sigma_{1}$ is smaller than the other two, the shape of the stress tensor would be the oblate spheroid of figure $2 \mathrm{c}$ ). This gives the following relationship between the principal values, invariants, and shape.

$$
\begin{aligned}
& \lambda_{1}>0 \quad \Rightarrow \quad I_{3}>0 \Rightarrow \text { Rod-like turbulence } \\
& \lambda_{1}<0 \quad \Rightarrow \quad I_{3}<0 \Rightarrow \text { Disk-like turbulence }
\end{aligned}
$$

Eliminating $\lambda_{1}$ from the relations in 22 we get

$$
I_{3}=A\left[2\left(-\frac{I_{2}}{3}\right)^{3 / 2}\right]
$$

The term in the parenthesis will always be greater than zero since $I_{2} \leq 0$ (see equation (7)), so the sign of $I_{3}$ will be determined by $A$. Comparing this with the statements 23 and $24, A$ will have the following values

$$
A=\left\{\begin{array}{rll}
1 & , & \text { Rod-like turbulence } \\
-1 & , & \text { Disk-like turbulence }
\end{array}\right.
$$

With respect to the invariant map of Lumley and in terms of the shape of the stress tensor this gives the designation of axes shown in figure 3 .
The error in the designation encountered in articles and textbooks is that the two axisymmetric limits with rod like and disk like turbulence are switched because some researchers talk about the shapes of the turbulent eddies rather than those of the stress tensor. In terms of vorticity the visualization of the turbulence would be opposite to that of the stresses. This may be exemplified by the flow through an axisymmetric contraction as shown in figure 4 . Figure $4 \mathrm{~A}$ ) illustrates a streamwise vortex tube before and after a contraction. For an incompressible fluid the transport equation for vorticity is given by the Helmholtz equation

$$
\frac{D \boldsymbol{\omega}}{D t}=(\boldsymbol{\omega} \nabla) \boldsymbol{U}+v \nabla^{2} \boldsymbol{\omega},
$$

where $\mathbf{U}$ is the fluid velocity and the vorticity $\boldsymbol{\omega}$ is defined by the curl of the velocity

$$
\boldsymbol{\omega}=\nabla \times \mathbf{U} .
$$

The first term on the right of (26) is called the vortex stretching term whereas the second term is a viscous diffusion term. Through the contraction the streamwise component of the vorticity $\omega_{1}=$ is increased by vortex stretching term since it includes the term $\omega_{1} \partial U_{1} / \partial x_{1}$. $\partial U_{1} / \partial x_{1}$ is positive due to the decrease in flow area and therefore a streamwise vortex tube is stretched and obtains a rod-like shape after the contraction. At the same time, due to the continuity requirement $\nabla \mathbf{U}=0$, the vorticity in the directions normal to the streamwise direction, $\omega_{2}$ and $\omega_{3}$, are attenuated.

For the turbulent stress tensor, on the other hand, the production of the streamwise turbulence component is suppressed by the secondary production term $-\tau_{11} \partial U_{1} / \partial x_{1}$ (see [4]). $\tau_{11}$ is always positive and since the streamwise gradient is positive the production term will become negative. The result is that the stress ellipsoid, according to the analysis in the previous section, becomes flattened in the streamwise direction and obtains a disk-like shape as illustrated in figure 4B).

\section{Summary}

The aim of the current paper is to clarify the terminology used when the axisymmetric limits of the Lumley triangle are related 


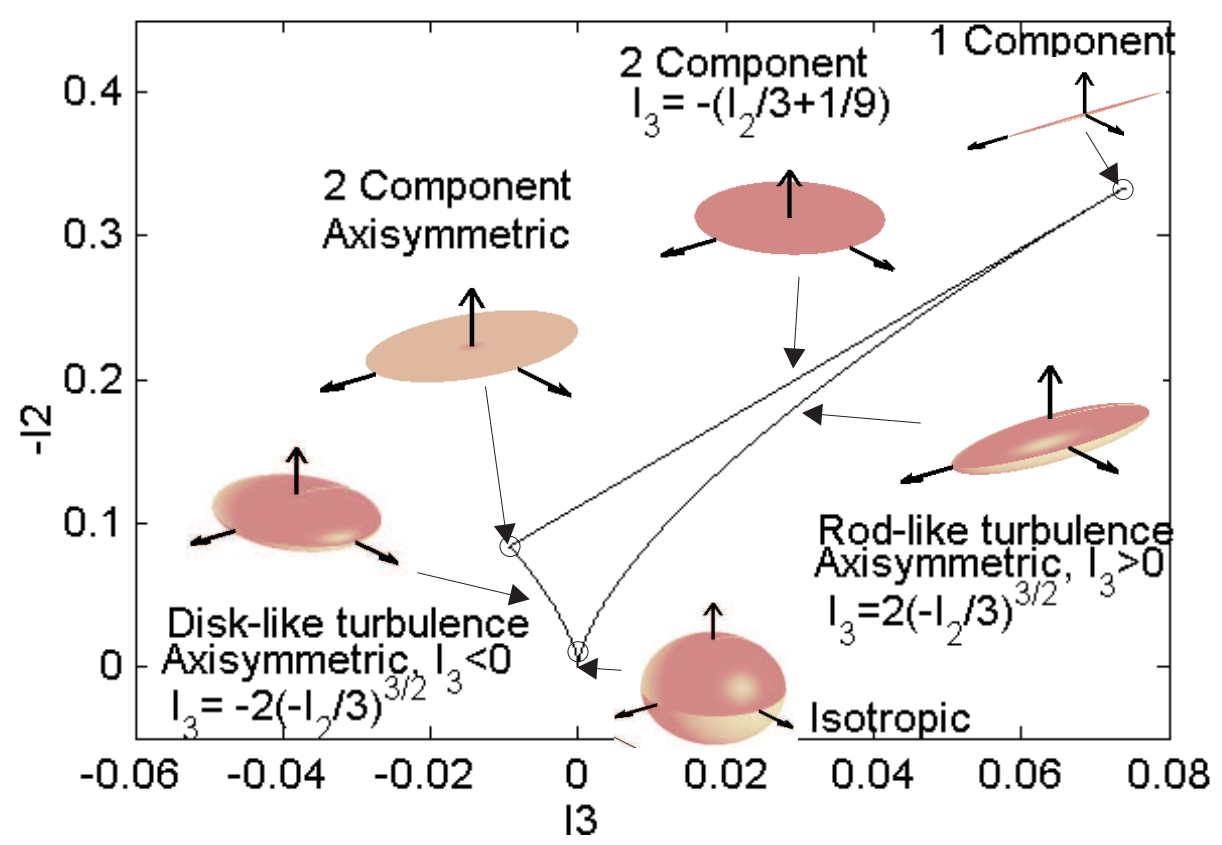

Figure 3: Anisotropy invariant map with correct designation of the axisymmetric states.
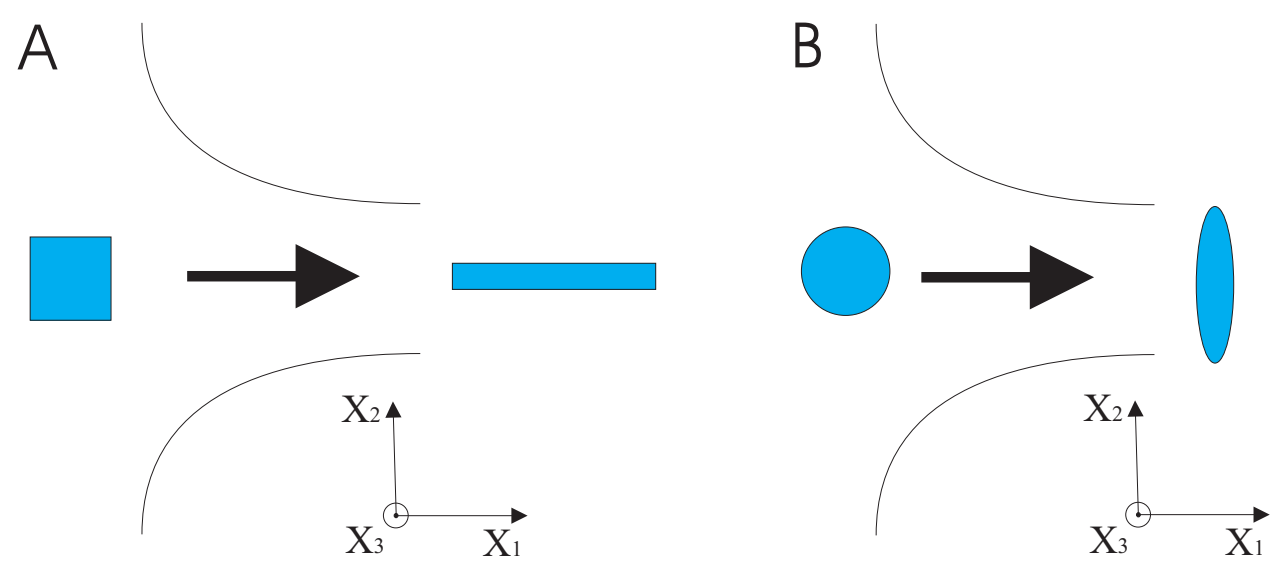

Figure 4: Illustration of streamwise vortex tube stretching(A) and stress ellipsoid deformation(B).

to shapes of the turbulence. As demonstrated by the above discussion the shape designated to the axisymmetric limits in the invariant map may be interpreted in two ways. Although this has no effect on turbulence modelling it does lead to some confusion when visualizing the turbulence. Since the invariant map itself is based on the turbulent stress tensor, it would be preferable that the designation of its axis is related to this quantity only.
[4] Torbergsen, L., Experiments in turbulent pipe flow, Ph.D. thesis, Norwegian University of Science and Technology, 1998.

\section{References}

[1] Choi, K. and Lumley, J., The return to isotropy of homeneous turbulence, J. Fluid Mech., 436, 2001, 59-84.

[2] Lee, M. and Reynolds, W., Numerical experiments on the structure of homogeneous turbulence, Technical Report Rep. TF-24, Thermoscience Division, Stanford University, 1985.

[3] Lumley, J., Computational modelling of turbulent flows, Adv.Appl.Mech., 18, 1978, 123-176. 